\section{Estudo \\ crobate}

em Cestão

Planejamento
Revista Estudo \& Debate, Lajeado, v. 27, n. 3, 2020. ISSN 1983-036X

DOI: http://dx.doi.org/10.22410/issn.1983-036X.v27i3a2020.2431

\title{
DIMENSIONAMENTO DA FORÇA DE TRABALHO DA SECRETARIA DE ESTADO DA SAÚDE PÚBLICA DO RIO GRANDE DO NORTE
}

\author{
Aline da Silva Rocha ${ }^{1}$, Themis Cristina Mesquita Soares ${ }^{2}$, Francisca Joice Souza Silva ${ }^{3}$, \\ Maria Dianna Souza ${ }^{3}$, Sara Taciana Firmino Bezerra ${ }^{4}$, José Giovani Nobre Gomes ${ }^{4}$
}

\begin{abstract}
Resumo: Problema de pesquisa: o perfil da força de trabalho da Secretaria de Saúde Pública do Estado do Rio Grande do Norte e o déficit de servidores por cargo. Objetivo: descrever o dimensionamento da Força de Trabalho (FT) da Secretaria de Estado da Saúde Pública do Rio Grande do Norte. Método: foi realizado estudo com predomínio quantitativo, exploratório a partir de dados secundários. As fontes de dados foram os sistemas de banco de dados da Coordenadoria de Recursos Humanos da Secretaria Estadual de Saúde Pública do RN e Planilhas de déficit de servidores por cargo, analisados estatisticamente e apresentados em gráficos e tabelas. Resultados: a FT da SESAP/RN encontra-se com perfil predominantemente envelhecido, feminino, e com tempo de serviço igual ou superior a 25 anos. Constatou-se alto índice de vacâncias, principalmente por aposentadorias, que comprometem a manutenção de servidores nas escalas de plantão; a prática de desvios de função nos diferentes níveis; e elevado déficit de servidores. Com relação a ferramentas tecnológicas utilizadas como instrumento de gestão da FT, verificou-se que se mostram superficiais, desatualizadas, com informaçóes divergentes, e que náo vêm atendendo a demandas e processos, como o dimensionamento de RH, feito manualmente em planilhas de Excel, suscetível a erros. Conclusão: há necessidade de sistema de informação de dimensionamento para a coleta e visualização do quantitativo que venha a gerir e monitorar a FT da SESAP/ RN.
\end{abstract}

Palavras-chave: Saúde do Trabalhador; Downsizing Organizacional; Emprego; Organização e Administração.

1 Enfermeira. Especialista em Gestão do Trabalho e da Educação na Saúde (PROGESUS) Servidora da Coordenadoria de Recursos Humanos da SESAP/RN.

2 Educadora Física. Docente adjunta da Universidade do Estado do Rio Grande do Norte (UERN). Docente do Programa de Mestrado Acadêmica em Planejamento e Dinâmicas Territoriais do Semiárido (PLANDITES).

3 Graduandas do curso de Enfermagem da UERN.

4 Enfermeiros. Docentes adjuntos da Universidade do Estado do Rio Grande do Norte (UERN). Docentes do Programa de Mestrado Acadêmica em Planejamento e Dinâmicas Territoriais do Semiárido (PLANDITES). 


\title{
DIMENSIONING OF THE WORKFORCE OF THE SECRETARY OF STATE OF PUBLIC HEALTH OF RIO GRANDE DO NORTE
}

\begin{abstract}
Research problem: the profile of the workforce of the Rio Grande do Norte State Department of Public Health and the deficit of employees by position. Objective: to describe the size of the Work Force of the State Department of Public Health of Rio Grande do Norte. Methods: predominantly quantitative, exploratory study was performed based on secondary data. The data sources were the database systems of the Coordination of Human Resources of the State Secretariat of Public Health of the RN and Servers deficit charts by position, analyzed statistically and presented in graphs and tables. Results: The SESAP / RN workforce is aged, female, and has an equal and / or longer service life of 25 years. The FT of SESAP / RN has a profile predominantly aged, female, and has equal and / or longer service life of 25 years. It was observed a high index of vacancies, mainly of retirements, which compromise the maintenance of servers in the call scales, the practice of deviations of function in the different levels, high server deficit. With regard to technological tools used as a tool for the management of the Work Force, it has been found that they are superficial, out of date, with divergent information, and that does not meet demands and processes, such as HR sizing, done manually in worksheets of Excel, susceptible to errors. Conclusion: there is a need for a sizing information system for the collection and visualization of the quantity that will manage and monitor the SESAP / RN workforce.
\end{abstract}

Keywords: Occupational Health; Personnel Downsizing, Employment, Organization and Administration.

\section{INTRODUÇÁO}

Gerir e organizar a saúde pública no Brasil não vem sendo tarefa fácil. Dentre os seus principais problemas estão a falta e/ou desigualdade no acesso aos serviços de saúde, a superlotação das urgências e emergências, a escassez de profissionais e de material nas unidades de saúde, a falta de leitos hospitalares e demora na realizaçáo de serviços de apoio diagnóstico, as quais estão fragilizando o Sistema Único de Saúde (SUS). Este pode ser tratado como uma preocupação ligada ao ramo da economia da saúde, cujo objetivo é garantir, de forma eficaz o direito ao acesso integral à saúde para toda a população brasileira (MORAES et al, 2019).

Algumas iniciativas foram tomadas para minimizar tais problemas, dentre eles merece destaque a criação dos Princípios e Diretrizes para a Gestão do Trabalho no SUS, que "trouxe orientaçôes fundamentais para a organização de processos gerenciais, como os relacionados à Política de Recursos Humanos no setor público do SUS" (NOGUEIRA, 2017).

Para a superação desse quadro, "precisam ocorrer mudanças em múltiplas dimensóes: políticas, ideológicas, jurídico-legais e organizacionais no nível macro, mas, também, no processo de trabalho, nas relaçōes pessoais e nas práticas individuais de saúde, no nível micro" (NUNES et al., 2016).

"A definição de responsabilidades entre os gestores configura um processo de organização de um sistema de governança no âmbito do SUS, voltado para a efetiva regulação do sistema, em especial para a organização da oferta e da demanda em uma de suas áreas mais sensíveis" (BARBOSA et al, 2016).

Dentre as principais atribuições na área de Gestão do Trabalho, está a de elaborar um diagnóstico da situação da Força de Trabalho (FT) que inclua aspectos como quantidade de trabalhadores por cargo, o seu perfil, os cedidos de outras esferas de gestão e os terceirizados; 
local de lotação; distribuição dos profissionais, comparando-os com o tamanho da população usuária e por categorias profissionais e especialidades. "Inclui-se também o de elaborar quadro de necessidades de trabalhadores para a manutenção da estrutura gestora e realizar concurso público para contratar e manter o quadro de pessoal necessário à execução da gestão e das açôes em serviços de saúde" (SOARES et al., 2016).

Dessa forma, a FT torna-se componente primordial para potencializar resultados organizacionais um estudo sobre a estrutura organizacional, o perfil da FT, os produtos e serviços ofertados e a tecnologia envolvida, ou seja, conhecer a FT e seu perfil tornase imprescindível para a realização de um planejamento estratégico visando a tomada de decisôes gerenciais eficientes a curto, médio e longo prazos.

No contexto da Coordenadoria de Recursos Humanos da Secretaria Estadual de Saúde Pública do RN (SESAP/RN), há necessidade constante de informaçôes sobre a situação da FT, pelo enfrentamento frequente de entraves, distorçóes, duplicidade de dados, sistemas de informaçóes sem cruzamento de dados e dinamicidade, que interferem no processo de trabalho e geram conflitos de informaçóes que podem interferir num correto dimensionamento da FT da secretaria.

Diante dessa problemática, tornou-se imprescindível um estudo do perfil da FT para minimizar possíveis erros e distorçóes no dimensionamento de pessoal das unidades de saúde.

Espera-se, com este trabalho, subsidiar o estabelecimento de parâmetros e estratégias para a tomada de decisão, referente à movimentação e relotação de servidores, e provisão de vagas para concurso público, garantindo o pleno funcionamento dos serviços em saúde, proporcionando uma otimização dos gastos.

Assim, o objetivo deste trabalho é descrever e analisar a FT da Secretaria Estadual de Saúde Pública do RN, buscando apresentar o perfil dos servidores da SESAP e conhecer o déficit de servidores por cargo.

\section{REFERENCIAL TEÓRICO}

Quando se discute a hegemonia das organizaçóes, percebe-se que se pode explicá-las por meio da escolha e posicionamento de um determinado setor, pelo desenvolvimento de recursos valiosos e não imitáveis ou ainda por rotinas e capacitaçôes dinâmicas (BRITO; OLIVEIRA, 2016).

Gestão em administração pública se faz para servir os cidadãos e usuários de um serviço de saúde, por exemplo. Porém, servir o público e administrá-lo não é a mesma coisa, o último pode ter uma conotação de gerenciar a si próprio, o primeiro já tem o seu foco em atender as necessidade da populaçáo a que ele serve. No serviço público, como no serviço de saúde, é necessário atuar em horário adequado, ser afável no tratamento com as pessoas, ter celeridade, ser eficaz, ser profissional, entre outros atributos. Visando isso, o sistema precisa estar bem organizado para atuar nos problemas e encontrar soluçóes, a partir de uma boa gestão pública (MOZZICAFREDDO, 2017). 
O contexto atual se caracteriza por sua dinamicidade, tudo se modifica rapidamente, fazendo com que as organizaçóes se antecipem a essas mudanças. Mudanças essas que decorrem de um novo mundo de negócios cuja competitividade muitas vezes se torna a marca (MALAFAIA, 2017).

A inserção do trabalhador no sistema de saúde, remete a compreensão da complexidade no mercado de trabalho da saúde, além dos empregos formais e informais existentes, bem como os postos de trabalho ocupados, a tendência de se comprometerem com vários riscos, às condiçóes de trabalho e o processo de adoecimento e como se dá a formação e a educação permanente e suas repercussóes no processo de trabalho individual e coletivo (MACHADO; XIMENES NETO, 2018)

Um dos temas com maior destaque no campo da estratégia empresarial diz respeito aos fatores de desempenhos das organizaçóes sociais. Recursos humanos se destacam como raros à medida que detém conhecimento pertinente, construído por meios sociais complexos (BRITO; OLIVEIRA, 2017).

Com isso, nota-se a necessidade de uma notória atenção por parte dos gestores e instituiçóes para a adequação entre os objetivos institucionais e as necessidades apresentadas pela população usuária. Torna-se evidente que pensar em gestão do trabalho como política significa pensar estrategicamente, buscando sempre a defesa de requisitos básicos para a maior valorizaçáo do profissional e do seu trabalho como, carreiras, salários, capacitaçáo e educação permanente entre outros (MACHADO; XIMENES NETO, 2018).

O enfoque na melhoria da gestão pública, buscando maior eficiência, levou a reforma da Lei n. ${ }^{\circ}$ 8666/93 (Licitaçôes) e, também, com a Lei n. ${ }^{\circ}$ 101/00 (Responsabilidade Fiscal). Esses dois marcos legais interferem na gestão e no planejamento ao introduzir parâmetros de transparência, controle e responsabilidade (ANDRADE, 2015). Com essas açóes, procurou-se melhorar a gestão pública.

Entretanto, a administração pública está imersa em um contexto complexo, o qual evidencia a não funcionalidade plena no seu desempenho. Além disso, percebe-se uma relativa inadequação ao tentar fazer cumprir a sua funçấo de serviço público. A gestáo se encontra de frente ao desafio de modernização de sua ação. Nisso, a administração deve melhorar para responder aos problemas e, assim, fortalecer a confiança dos cidadãos (MOZZICAFREDDO, 2017).

Para cumprir as suas funçóes, a gestão pública, deve observar a sua atuação e elaborar estratégias e critérios para o atendimento das demandas sociais, o alcance da eficiência, eficácia e efetividade dos serviços. Para tal, é preciso trabalhar no seu gerenciamento organizacional a fim de otimizar a utilização dos recursos despendidos para tal. A administração pública deve empregar os recursos adequadamente e, ao mesmo tempo, oferecer um melhor atendimento às necessidades (MESSIAS et al, 2018).

$\mathrm{Na}$ área da saúde, um aspecto bastante relevante trata da sua FT, na medida em que é necessário se adequar às transformaçóes do mundo do trabalho, sendo imprescindível que o setor público acompanhe a evolução na área de produção tecnológica. Além disso, a inserção e aquisiçáo de novas competências, de formação técnica e acadêmica são requisitadas aos trabalhadores e gestores, implicando em uma nova redefiniçấo de selecionar, recrutar e 
qualificar os profissionais em saúde, processo esse dinâmico e contínuo, merecendo um estudo profundo e sistemático na FT em saúde, para garantir uma gestáo e um atendimento de qualidade à população (LACOMBE, 2017).

Por outro lado, o dimensionamento inadequado ou insuficiente dos profissionais de saúde e de enfermagem pode estar associado com o aumento de doenças ocupacionais, ocorr6encia de eventos adversos e insatisfaçáo no usuário no atendimento (BRANDÃO et al, 2017).

Além disso, há necessidade de organizar um banco de dados sobre os trabalhadores do SUS, que facilite uma avaliação permanente da necessidade de servidores para realizaçâo de açôes e serviços de saúde. "Esse banco de dados deverá ser atualizado sistematicamente para ser utilizado no planejamento e implementação do Sistema de Saúde, nas açóes de administração, de desenvolvimento dos trabalhadores e de controle social da Gestão do Trabalho no SUS" (NOGUEIRA, 2017).

O Ministério da Saúde, nas últimas décadas vem realizando estudos e desenvolvendo estratégias na área de recursos humanos (BRASIL, 2005), em conformidade com organismos internacionais como a Organização Pan-Americana da Saúde e Organização Mundial de Saúde que enfatizam a importância da força de trabalho no desenvolvimento e fortalecimento dos sistemas de saúde pública.

Portanto, entende-se que o dimensionamento de pessoal permite a qualidade do processo de tomada de decisão no planejamento em saúde, na medida em que dá o suporte de caracterização do cenário dos profissionais, sua distribuição, movimentaçãa, déficits e superávits, articulando essas informaçôes com as necessidades da população que goza do SUS (RAMOS; POSSA, 2016).

\section{METODOLOGIA}

Trata-se de uma pesquisa predominantemente quantitativa do tipo exploratória, realizada junto à SESAP/RN. Atualmente em processo de descentralização, a secretaria possui oito regióes de saúde, as quais contemplam 24 (vinte e quadro) unidades hospitalares, 06 (seis) unidades regionais administrativas, 03 (três) unidades de agentes terapêuticos, 05 (cinco) Hemocentros, 04 (quatro) laboratórios, 01 (um) Banco de Leite, 01(um) Centro de Reabilitação Infantil e Adulto, 01 (um) Centro de Formação de Pessoal para os Serviços de Saúde, 01 (um) Serviço de Verificação de Óbitos, 01 (um) Central do SAMU Metropolitano e o Nível Central.

Foram utilizados como fonte de informação, dados secundários sistemas de banco de dados da Coordenadoria de Recursos Humanos da Secretaria Estadual de Saúde Pública do RN (Sistema ERGON e Portal RH) e Planilhas de déficit de servidores por cargo da Comissão Técnica de Dimensionamento de RH.

Os dados foram coletados em 2016 e foram organizados utilizando o software Excel, passando por análise estatística descritiva e estão apresentados em gráficos e tabelas que serviram para análise e posterior conclusóes. 
Como trata-se de pesquisa com dados secundários, a mesma não passou por apreciação em Comitê de Ética em Pesquisa.

\section{RESULTADOS E ANÁLISE/DISCUSSÓES}

A SESAP/RN possuía até maio de 2016, 14.974 servidores, dos quais 1.070 encontravam-se trabalhando em unidades municipais de saúde do Estado; os demais 13.904 trabalhando nas unidades estaduais (Tabela 1).

TABELA 01 - Distribuição da Força de Trabalho da SESAP/RN nas regiōes de saúde do estado do RN

\begin{tabular}{l|r|r|r|r|r|r}
\hline & $\begin{array}{r}\text { Servidores em Unidades } \\
\text { estaduais }\end{array}$ & \multicolumn{2}{|c}{$\begin{array}{c}\text { Servidores Cedidos a } \\
\text { municípios }\end{array}$} & \multicolumn{2}{c}{ TOTAL } \\
& $\mathrm{N}$ & \multicolumn{1}{|c|}{$\begin{array}{r}\text { \% } \\
\text { Regiáo }\end{array}$} & $\mathrm{N}$ & \multicolumn{1}{c}{$\%$} & $\mathrm{~N}$ & $\%$ \\
Metropolitana & 8857 & 63,7 & 561 & 52,4 & 9.418 & 62,9 \\
URSAP I & 404 & 2,9 & 57 & 5,3 & 461 & 3,1 \\
URSAP II & 2336 & 16,8 & 169 & 15,8 & 2.505 & 16,7 \\
URSAP III & 291 & 2,1 & 55 & 5,1 & 346 & 2,3 \\
URSAP IV & 945 & 6,8 & 49 & 4,6 & 994 & 6,6 \\
URSAP V & 334 & 2,4 & 51 & 4,8 & 385 & 2,6 \\
URSAP VI & 737 & 5,3 & 128 & 12,0 & 865 & 5,8 \\
TOTAL & 13.904 & 100,0 & 1.070 & 100,0 & 14.974 & 100,0 \\
\hline
\end{tabular}

Fonte: CRH-SESAP-RN, 2016.

A FT da SESAP/RN é composta por profissionais de nível elementar, médio e superior, terceirizados, cooperativas, cargos comissionados, cedidos, redistribuídos e requisitados. Mas, o objeto de estudo foi apenas os servidores de cargo efetivo. Estes foram agrupados e distribuídos a partir de 2006 de acordo com o Plano de Cargos, Carreiras e Remuneraçóes (PCCR) (Tabela 2). 
TABELA 02 - Distribuição da Força de Trabalho por sexo, faixa etária, escolaridade, carga horária e tempo de serviço. Natal-RN, 2016

\begin{tabular}{|c|c|c|}
\hline $\begin{array}{l}\text { Variáveis } \\
\mathrm{N}=14.974\end{array}$ & $\mathbf{N}$ & $\%$ \\
\hline \multicolumn{3}{|l|}{ Sexo } \\
\hline Feminino & 10.216 & 68,22 \\
\hline Masculino & 4.758 & 31,78 \\
\hline \multicolumn{3}{|l|}{ Faixa etária } \\
\hline $19-29$ & 604 & 4,0 \\
\hline $30-39$ & 2.993 & 20,0 \\
\hline $40-49$ & 3.439 & 23,0 \\
\hline $40-59$ & 6.058 & 40,4 \\
\hline $60-69$ & 1.851 & 12,4 \\
\hline 70 ou mais & 29 & 0,2 \\
\hline \multicolumn{3}{|l|}{ Escolaridade } \\
\hline Fundamental incompleto & 325 & 2,2 \\
\hline Fundamental completo & 2.546 & 17,0 \\
\hline Médio completo & 6.341 & 42,3 \\
\hline Superior & 5.649 & 37,7 \\
\hline Pós-Graduação & 113 & 0,8 \\
\hline \multicolumn{3}{|l|}{ Carga horária } \\
\hline 40 horas & 2.087 & 13,94 \\
\hline 30 horas & 12.060 & 80,54 \\
\hline 24 horas & 150 & 1,0 \\
\hline 20 horas & 677 & 4,52 \\
\hline \multicolumn{3}{|l|}{ Tempo de serviço (anos) } \\
\hline $0-4$ & 2.553 & 17,0 \\
\hline $5-9$ & 2.538 & 17,0 \\
\hline $10-14$ & 119 & 0,8 \\
\hline $15-19$ & 1.004 & 6,7 \\
\hline $20-24$ & 1.916 & 12,8 \\
\hline $25-29$ & 3.445 & 23,0 \\
\hline $30-34$ & 2.826 & 18,9 \\
\hline 35 ou mais & 573 & 3,8 \\
\hline
\end{tabular}

Fonte: Sistema ERGON, CRH-SESAP-RN, 2016.

Entre os profissionais da área médica, 13,94\% possuem a carga horária de 40 horas semanais. Os técnicos em radiologia com 24 horas semanais correspondem a 1,0\%. Os 
que possuem a carga horária de 20 horas $(4,5 \%)$ são os profissionais Cirurgióes Dentistas, Veterinários e Médicos com redução de carga horária. E com 30 horas semanais 80,54\%, os demais profissionais da assistência, área administrativa e apoio de níveis superior, médio e elementar.

Fatores como falecimentos, exoneraçóes e aposentadorias, têm total relação e interferência na manutenção e aumento do déficit da FT (Tabela 03).

TABELA 03 - Distribuição da Vacância no período de 2009 a maio de 2016 na Secretaria de Saúde do Estado do Rio Grande do Norte. Natal-RN, 2016

\begin{tabular}{lrrrrrrrrrrrrr}
\hline \multicolumn{10}{c}{ Anos } \\
\hline \multicolumn{10}{c}{$\mathbf{2 0 0 9 - 2 0 1 0}$} & \multicolumn{10}{c}{$\mathbf{2 0 1 1 - 2 0 1 2}$} & \multicolumn{2}{c}{ 2013-2014 } & \multicolumn{2}{c}{ 2015-2016 } \\
\hline Escolaridade & Elementar & Médio & Superior & Elementar & Médio & Superior & Elementar & Médio & Superior & Elementar & Médio & Superior \\
\hline Falecidos & 64 & 23 & 20 & 61 & 20 & 19 & 62 & 26 & 32 & 34 & 13 & 6 \\
\hline Exonerados & 21 & 39 & 55 & 11 & 15 & 52 & 31 & 77 & 125 & 15 & 43 & 66 \\
\hline Aposentados & 343 & 111 & 159 & 486 & 184 & 297 & 500 & 216 & 451 & 389 & 187 & 382 \\
\hline TOTAL & 835 & & & 1145 & & & 1520 & & 1135 & \\
\hline
\end{tabular}

Fonte: Sistema ERGON, CRH-SESAP-RN, 2016.

Quando relacionamos o quantitativo de servidores nomeados nos concursos públicos dos anos de 2008 e 2010, tem-se 5.091 servidores. Porém, foram geradas 4.635 vacâncias de profissionais de níveis médio e superior.

A partir dos resultados apresentados, constata-se que a maioria da FT concentra-se na região metropolitana de Natal com $64 \%$, o que reflete uma realidade histórica brasileira de aglomeração dos seus serviços nas grandes cidades. É consequência da própria estruturação capitalista que transformou as capitais em locais de acumulação e pontos estratégicos para o desenvolvimento regional (SOARES, 2018). Em segundo lugar, encontra-se a II URSAP com $17 \%$.

Há ainda, 1.070 funcionários (7,4\%) que pertencem a SESAP/RN, mas estão no momento municipalizados e desempenhando suas funçóes em unidades municipais de saúde.

Constata-se a existência de cargos criados no PCCR, cujas vagas ainda estão em aberto. Por outro lado, verifica-se que cargos como o de Biólogo já está bem próximo de atingir o seu limite e no caso dos técnicos em radiologia, o limite já ultrapassou. Isso reflete a necessidade de rever a lei, assim como as necessidades dos cargos e de suas respectivas vagas.

Essa necessidade se reflete também pela expansão e criação de serviços que demandam novos cargos e atribuiçóes, bem como a exclusão de outros que não estejam sendo utilizados. Infelizmente, inexiste um grupo ou comissão direcionado a rever o PCCR da Secretaria.

Os cargos com maior número de servidores são Auxiliares de Saúde 30,9\%, Técnicos em Enfermagem 20,7\%, Médicos 14,6\%, Enfermeiros 9,5\%, Farmacêuticos Bioquímicos 3,3\% e Cirurgióes Dentistas 2,9\%. Merece destaque a situação dos auxiliares de saúde, uma vez que 1/3 da FT é composta por profissionais que desempenham funçóes nas áreas de nutrição, lavanderia, manutenção, administrativo e apoio na assistência. A gestão tem 
reposto as vagas desses trabalhadores com a terceirização, uma vez que não se visualiza vagas para essas funçôes em concurso público. Outro destaque é a área de enfermagem com $30,2 \%$.

Mais de 2/3 da FT da SESPA/RN é composta por mulheres, o que reflete uma realidade vivenciada no mundo do trabalho em saúde, onde a presença feminina é majoritária, processo que chamamos de feminização (SILVA, SILVA e QUISSI, 2018). Um dos impactos dessa FT são os afastamentos para licença maternidade que acabam por interferir no dimensionamento de pessoal, gerando a necessidade de pagamento de plantóes extras (eventuais) para suprir o déficit deixado.

Os dados presentes no sistema ERGON não refletem a realidade da carga horária total de muitos servidores, uma vez que como exemplo, um servidor técnico em enfermagem que foi admitido para cumprir uma carga horária de 30 horas semanais (cerca de 108 horas mensais), pode possuir uma jornada especial que o leve a cumprir uma carga horária mensal de 144 horas a 192 horas.

A FT encontra-se envelhecida, uma vez 53\% dos servidores têm idade igual e/ou superior a 50 anos. Dessa forma, mais da metade da FT da SESAP/RN, encontra-se em um período da vida em que a mente e o corpo já começam a desacelerar, como consequências de hábitos pouco saudáveis ou mesmo do processo de trabalho em saúde que começam a refletir no desempenho e produtividade (COSTA; SOARES, 2018). Isso interfere de forma direta ou indireta no processo e manutenção da FT, já que o índice de adoecimento aumenta, intervindo na manutenção e no atendimento à população.

O nível de escolaridade é referente ao período de admissão. Portanto, são números que não refletem a atual situação de escolaridade dos servidores, uma vez que o próprio governo estadual oferece cursos de formação técnica, superior e pós-graduação.

Sobre a situação de tempo de serviço da FT da SESAP, observa-se que 22,7\% dos servidores possuem 30 anos ou mais de dedicação. Somando os servidores que estão na faixa dos 25 a 29 anos de tempo de serviço, que em muitos casos averbam seu tempo de serviço insalubre e as licenças prêmios náo gozadas para aumentar o tempo de serviço, esse percentual pode chegar até $45,71 \%$, ou seja, quase metade da FT da SESAP até nos próximos 05 anos deverá estar aposentada. Esse fato pode ser mais crítico no caso das mulheres, uma vez que um dos requisitos para a aposentadoria é ter no mínimo 30 anos de efetivo exercício, e no caso dos homens 35 anos de exercício. Isso pode gerar um colapso no atendimento à população, caso o quantitativo não seja reposto.

Entre o ano de 2009 até maio de 2016, 380 servidores faleceram, 3.705 se aposentaram e 580 foram exonerados. As exoneraçóes estáo relacionadas a servidores que respondiam a processos de sindicância administrativa, acumulação de cargos, abandono de cargo e por motivo de nomeação em outro concurso público, destacando-se os servidores de nível superior pertencentes à categoria médica.

Houve a geração de 4.635 vacâncias entre os anos de 2009 a maio de 2016 com o maior percentual de vacâncias entre os profissionais de nível elementar. Nesse período, houve um acréscimo de 456 servidores na FT da SESAP/RN, considerando as nomeaçóes e as vacâncias geradas. Ou seja, o déficit de servidores continua persistindo, uma vez que 
o incremento na FT foi só o necessário para repor as vacâncias, não suprindo o déficit de pessoal.

Outro fator bastante pertinente quanto ao levantamento de déficit de profissionais da SESAP, é a presença de desvios de função nas diferentes categorias profissionais, considerado como atribuiçóes desempenhadas pelo servidor que não são inerentes ao seu cargo de origem. É prática proibida no serviço público pela Lei Complementar No 122, de 30 de Junho de 1994, que dispóe sobre o Regime Jurídico Único dos servidores públicos civis do Estado do Rio Grande do Norte, em seu Título I, Capítulo Único das Disposiçóes Preliminares, Art. $3^{\circ}$, Inciso II sobre as vedaçóes (RIO GRANDE DO NORTE, 1994).

Podem ser várias as causas do desvio de função, dentre elas a terceirização de serviços de limpeza, manutenção e apoio, obrigando os servidores a desempenhar funções em outras áreas; a falta de pessoal especializado em determinadas funçóes; a continuidade dos estudos e posterior interesse dos servidores em mudar de função, por muitas vezes achar a função inicialmente exercida ser considerada inferior (PASSOS; NOGUEIRA, 2018).

\section{CONCLUSÃO}

Ao longo do estudo, ficou claro que a FT da SESAP/RN concentra-se em sua maioria na região metropolitana, com perfil predominante de mulheres. São 14.974 servidores concursados, cuja faixa etária chega a $53 \%$ de servidores com idade igual ou superior a 50 anos. Isso mostra uma FT envelhecida, cujo tempo é igual e/ou superior a 25 anos de serviços prestados. Conclui-se ainda que em curto prazo, a SESAP poderá perder quase metade da sua atual FT, tornando imprescindível a urgente realização de certames para contratação de servidores para minimizar tal crise.

Alguns dos fatores encontrados que interferem no processo de gestão da FT foram as exoneraçōes, falecimentos e principalmente aposentadorias, gerando uma vacância de 4.635 servidores. Durante este período, foram realizados dois concursos públicos que somaram 5.091 servidores nomeados. Consequentemente, o déficit só foi reduzido em apenas 456 servidores. Este valor pode ser agravado pelo fato de que a SESAP ainda não instituiu para o cálculo da FT um percentual como índice de segurança técnica, para os servidores afastados, de férias e de licença.

Com isso, vemos que o dimensionamento da FT não vem tendo a devida relevância e o reconhecimento como ferramentas de gestão em saúde, imprescindíveis para o planejamento e a efetivação da atenção, redução de custos e o monitoramento da gestão do trabalho.

Entende-se como limitação do trabalho que as informaçóes manuais para o levantamento do déficit estão em constante atualização, devido ao grande dinamismo das escalas, com relação a mudanças de setor, de lotação, servidores recém nomeados, afastamentos, licenças que não são monitoradas em tempo real. Esse fator prejudica a ação mais eficaz do dimensionamento.

Com isso, espera-se que o setor possa inovar e aderir a novas tecnologias para tornar o trabalho do dimensionamento de pessoal no estado mais efetivo, de acordo com as 
necessidades da população. Trabalhos que possa apresentar dados mais atuais são necessários para viabilizar a discussão mais aprofundada do tema.

\section{REFERÊNCIAS}

ANDRADE, EG. Diferentes estratégias de gestão de estabelecimentos de saúde vinculados ao Sistema Único de Saúde (SUS). Debates GV Saúde. Edição especial, p. 40-57, mar. 2015. Available from: https://www.google.com/url?sa=t\&rct=j\&$\mathrm{q}=\&$ esrc $=$ \&\&source=web\&cd=1 \&cad=rja\&uact=8\&ved=2ahUKEwjF1 qzcicflAhUwIbkGHftMD6gQFjAAegQIARAC\&url=http\%3A\%2F\%2Fbibliotecadigital.fgv. br\%2Fojs\%2Findex.php\%2Fdebatesgvsaude\%2Farticle\%2FviewFile\%2F76844\%2F73680\&usg=AOvVaw1lkR1PAK6yU0_nWrs_NEof

BARBOSA, DVS; BARBOSA, NB; NAJBERG, E. Regulação em Saúde: desafios à governança do SUS. Cad. saúde colet. [Internet], v. 24, n. 1, p. 49-54. 2016. Available from: http://www.scielo.br/scielo.php?script=sci_arttext\&pid=S1414462X2016000100049 \&lng=en. DOI: http://dx.doi.org/10.1590/1414$462 \times 201600010106$.

BRANDÃO, M. G. S. A. et al. Dimensionamento de Enfermagem como ferramenta de gestão do serviço de saúde. RETEP- Rev. Tendên. da Enferm. Profis., v. 9, n. 4, p. 23062310, 2017. Disponível em: http://www.coren-ce.org.br/wp-content/uploads/2019/02/ DIMENSIONAMENTO-DE-ENFERMAGEM-COMO-FERRAMENTA-DEGESTÃO-DO-SERVIÇO-DE-SAÚDE.pdf. Acesso em: 03/08/2020.

BRASIL. Ministério da Saúde. Conselho Nacional de Saúde. Princípios e diretrizes para a gestáo do trabalho no SUS (NOB/RH-SUS) / Ministério da Saúde, Conselho Nacional de Saúde. - 3. ed. rev. atual. - Brasília: Ministério da Saúde, 2005.

DE BRITO, Renata Peregrino; DE OLIVEIRA, Lucia Barbosa. A relação entre gestão de recursos humanos e desempenho organizacional. Brazilian Business Review, v. 13, n. 3, p. $94,2016$.

DE MALAFAIA, Gabrila Silva. Gestão estratégica de pessoas em ambientes multigeracionais. VII Congresso Nacional de Excelência em Gestáo, p. 1-13, 2017

COSTA, DGS; SOARES, N. Envelhecimento e velhices: heterogeneidade no tempo do capital. Serviço Social \& Realidade. [Internet.]; v. 25, n. 2, p. 57-67. 2018 Available from: https://ojs.franca.unesp.br/index.php/SSR/article/view/2519/2225

LACOMBE, FJM. Recursos Humanos: princípios e tendências. Editora Saraiva, São Paulo. 2017. 
MACHADO, Maria Helena; XIMENES NETO, Francisco Rosemiro Guimarães. Gestão da Educação e do Trabalho em Saúde no SUS: trinta anos de avanços e desafios. Ciência \& Saúde Coletiva, v. 23, p. 1971-1979, 2018.

MESSIAS, D. et al. Gestão de custos no setor público: um panorama de experiências internacionais. Rev. Serv. Público Brasília. v. 69, n. 3, p. 585-604, jul/set 2018. DOI: https://doi.org/10.21874/rsp.v69i3.2961

MORAES, M. X. et al. Influência dos acidentes de trânsitos em rodovias federais sobre os custos do Sistema Único de Saúde, no período de 2002 a 2012. Revista Estudo \& Debate, Lajeado, v. 26, n. 4, 2019. DOI: http://dx.doi.org/10.22410/issn.1983-036X. v26i4a2019.2178

MOZZICAFREDDO, J. Como Tornar a Administração Pública um Serviço Público no Contexto de Estados e Sociedades Complexas. Estudos da Administraçáo e Sociedade. v.2, n.1, p.20-28, 2017. DOI: https://doi.org/10.22409/eas.v2i2.65

NOGUEIRA, AJFM. Teoria do Conhecimento e Estudos Organizacionais para Além de um Único Caminho. Teoria e Prática em Administração. [Internet]. São Paulo; v. 7, n. 2, p. 237-240. 2017 DOI: http://dx.doi.org/10.21714/2238-104X2017v7i2-37244

NUNES, EFPA. et al. Trabalho gerencial em Unidades Básicas de Saúde de municípios de pequeno porte no Paraná, Brasil. Interface (Botucatu) [Internet]; v. 20, n. 58, p. 573-584. 2016. Available from: http://www.scielo.br/scielo. php?script=sci_arttext\&pid=S1414-32832016000300573 \&lng=en. DOI: http://dx.doi. org/10.1590/1807-57622015.0065.

PASSOS, Rachel Gouveia; NOGUEIRA, Cláudia Mazzei. O fenômeno da terceirização e a divisão sociossexual e racial do trabalho. Revista Katálysis, v.21, n.3, p.484-503, 2018. Available from: https://periodicos.ufsc.br/index.php/katalysis/article/view/198202592018v21n 3p484 DOI: https://doi.org/10.1590/1982-02592018v21n3p484

RAMOS, L.; POSSA, L. B. Dimensionamento da força de trabalho no SUS: o trabalho (e trabalhador) vivo no planejamento do cuidado em saúde. Saúde em Debate., v. 2, n. 1, p. 43-52. 2016. DOI: http://dx.doi.org/10.18310/2446-4813.2016v2n1p43-52

RIO GRANDE DO NORTE (Estado). Lei complementar estadual no122, de 30 de junho de 1994. Dispóe sobre o regime jurídico único dos servidores públicos civis do Estado e das autarquias e fundaçóes públicas estaduais. Diário Oficial do Estado em $1^{\circ}$ de julho de 1994. Palácio Potengi, Natal, RN, 1 jul 1994.

SILVA, Lucas Souza; DA SILVA, Renata Menezes; QUISSI, Victor Hugo. Terceirização como expressão da precarização das relaçóes de trabalho na FCT/UNESP: A feminização como (des) realização do trabalho. Geografia em Atos (Online), v. 2, n.7, p. 147-159, 
2018. Available from: http://revista.fct.unesp.br/index.php/geografiaematos/article/ view/6111 DOI: https://doi.org/10.35416/geoatos.v2i7.6111

SOARES, Mirelle Inácio, et al. Gerenciamento de recursos humanos e sua interface na sistematização da assistência de enfermagem. Enfermería Global, v.15, n.2 , p. 341-375, 2016. Available from: http://scielo.isciii.es/pdf/eg/v15n42/pt_administracion3.pdf

SOARES, Paulo Roberto Rodrigues. Metropolização, aglomeraçóes urbano-industriais e desenvolvimento regional no sul do Brasil. Cadernos Metrópole., v.20, n.41, p.15-34, 2018. Available from: https://revistas.pucsp.br/metropole/article/view/2236-9996.20184101. DOI: https://doi.org/10.1590/cm.v20i41.33088. 